\title{
The Paradox of Early Stage Rectal Cancer: More ReSeARCh in the Right Direction
}

\author{
Paolo Goffredo, MD (i), and J. Joshua Smith, MD, PhD, FACS \\ Colorectal Service, Department of Surgery, Memorial Sloan Kettering Cancer Center, New York, NY
}

To date, local excision (LE) for early stage rectal cancer is reserved for $\mathrm{T} 1$ tumors with favorable characteristics, including small size, non-circumferential involvement of the rectal wall, well-differentiated tumors, and no lymphovascular and/or perineural invasion. ${ }^{1}$ Unfortunately, the preoperative identification of appropriate LE candidates poses significant challenges due to the low accuracy of both MRI and endorectal ultrasound, further limiting the number of patients eligible for this approach. ${ }^{2,3}$ Therefore, because neoadjuvant chemoradiation is administered to stage II/III patients only, current guidelines recommend total mesorectal excision (TME) for the majority of stage I patients. ${ }^{1}$ However, TME exposes this subgroup to the inherent risks of radical resection, including anastomotic leak or permanent colostomy, wound dehiscence, bowel, urinary, and sexual dysfunction-all without the option for organ-preservation. ${ }^{4}$ In the setting of increased use of organ-sparing approaches for locally advanced rectal cancer (LARC) after neoadjuvant treatments, along with increased patient interest, and further bolstered by the recent success of a prospective watch \& wait (WW) strategy demonstrating up to 50\% 3-year organ preservation rates, we are facing a challenging paradox..$^{5-7}$ Nonetheless, over the past decade, several trials have investigated the expansion of inclusion criteria for LE in early stage (T1-2N0) rectal cancer through the addition of neoadjuvant chemoradiation, with promising results. ${ }^{8-11}$

(C) Society of Surgical Oncology 2021

First Received: 8 November 2021

Accepted: 11 November 2021;

Published Online: 30 November 2021

J. J. Smith, MD, PhD, FACS

e-mail: smithj5@mskcc.org
Conversely, even fewer data are available for the management of down-staged LARC patients with standard of care being TME. ${ }^{1}$

The preliminary data of the ReSARCh (Rectal Sparing Approach After Neoadjuvant Therapy in Patients with Rectal Cancer) trial published in this edition of Annals of Surgical Oncology are intriguing. ${ }^{12}$ While the use of WW protocols in selected patients is well-accepted, this study provides some new data on two important topics: (1) reallife experience with universal neoadjuvant treatment for all stages of rectal cancer, and (2) organ-preserving management based on response (e.g., ycT1). This prospective, multicenter observational study included 160 patients with cT1-4N0-2b rectal cancers located within $12 \mathrm{~cm}$ from the anal verge and good performance status. After MRI-based staging, neoadjuvant treatment in the form of fluoropyrimidine-based chemotherapy alone $(n=3)$, long course radiation alone $(n=14)$, or a combination of the two (total neoadjuvant therapy with consolidation chemotherapy; $n=$ 143) was initiated. A first restaging with repeat MRI, CT, physical exam, CEA levels, and proctoscopy was then performed at 8 weeks. Partial response (mCR) was defined as the presence of a scar $\leq 2 \mathrm{~cm}$, while clinical complete response (cCR) was characterized by the absence of palpable mass, any mucosal abnormalities at endoscopy, and no MRI positive lymph nodes. TME was recommended in cases of poor response; otherwise, a second thorough restaging was completed at 12 weeks. At that time, patients with $\mathrm{mCR}$ were managed with $\mathrm{LE}$, while those with $\mathrm{cCR}$ were offered either LE or WW. Following LE, TME was recommended if poor prognostic factors (ypT $\geq 2$, highgrade ypT1, positive margins, lympho-vascular or perineural invasion, and TRG $\geq 3$ ) were identified on pathologic examination. 
The authors should be congratulated on completing this pragmatic study which pushes the field forward. Noteworthy is the low number of grade III-IV complications (3.1\%) for the LE group. Additionally, there was a significant increase of cCR rates from 36 to $60 \%$ between the first and the second re-staging-this approach supports using time to allow the tumor to respond and has been shown to be important in patients with LARC using a total neoadjuvant therapy strategy. ${ }^{6,13}$ In the cCR patients in this trial, $24 \%$ were found to have a local regrowth at 2 years, which is in line with other reports. ${ }^{5,714}$ Finally, the authors confirmed the known challenges in predicting a ypT0-1 with poor accuracy and a specificity of $42 \%$. Among those with $\mathrm{mCR}$, only $49 \%$ were ypT0, while $27 \%$ had ypT $\geq 2$; whereas closer examination of the cCR patients demonstrated that $74 \%$ were ypT0 and $6 \%$ were ypT $\geq 2$. Nonetheless, ypT1 with favorable characteristics appeared to have short-term outcomes similar to ypT0. It will be of interest to see the results at longer follow-up in this nonselected cohort of patients. An approach based on response has indeed been shown feasible and safe in cT2-3Nx midlow rectal cancer by Rullier et al. in the GRECCAR 2 trial. ${ }^{9}$ After total neoadjuvant treatment, the authors randomized good responders to either LE or TME, with the option to cross over for those LE patients with unfavorable features. Interestingly, there was no difference in recurrence and survival rates between the two arms at the 3-year time point.

Some of the limitations of this current study are acknowledged by the authors and include the lack of randomization and the short follow-up times. More importantly, the cohort is heterogeneous both for neoadjuvant treatment and staging. While this heterogeneity provides practical, real-life experience with the management of rectal cancer, it may detract from the translation of these results into the clinic. Allowing 3 different preoperative schemes, radiation alone, chemotherapy alone, and chemoradiation with varying total radiotherapy doses, creates subgroups within each arm that are underpowered to extrapolate meaningful clinical data. Moreover, including all stages from cT1N0 to cT4N2b might compromise some of the potential advantages of organ-sparing approaches for early stage rectal cancers. In fact, among the 11 patients who agreed to completion TME after LE per-protocol, 1 (9\%) had positive mesorectal lymph-nodes (ypT2N1). Interestingly, 57\% of those patients who were recommended to receive completion TME refused it. Longer follow-up will provide further information regarding the natural history of partially treated disease. Finally, the reader is left with questions about quality of life (QoL) that the authors promise to address in a subsequent publication. QoL endpoints are of particular relevance in those patients undergoing LE after a neoadjuvant regimen that includes radiation, as shown by Stijins et al. in the CARTS trial, a phase II study, including cT1-3N0 rectal cancer and allowing LE for incomplete responders. ${ }^{15}$ Despite good oncologic outcomes, the CARTS trial demonstrated that $80 \%$ of the cohort experienced varying degrees of low anterior resection syndrome (LARS), highlighting the poor functional results of a combination of radiation with LE already reported in the GRECCAR 2 trial. ${ }^{9}{ }^{15}$ Similarly, in a comparison between cT2N0 rectal cancer patients undergoing neoadjuvant chemoradiation and LE from the ACOSOG Z6041 trial, and the upfront surgery arm of the Dutch TME trial, receiving a combination of radiotherapyLE was associated with lower health-related QoL. ${ }^{8,16,17}$

Currently, a few trials investigating WW in complete responders and LE following chemotherapy alone are ongoing. ${ }^{18,19}$ Results of these studies, including the ReSARCh trial, may provide nuanced guidance to address the paradox of early stage rectal cancer, balancing oncologic and functional outcomes. It is our hope that studies like this will generate additional hypotheses to stimulate novel trial designs to usher in more organ-preservation strategies for our patients with early stage rectal cancers.

DISCLOSURE Jesse Joshua Smith—served as a clinical advisor to Guardant Health Inc. (2019).

\section{REFERENCES}

1. NCCN. NCCN Clinical Practice Guidelines in Oncology: Rectal Cancer. 2021. Accessed January 2021, 2021.

2. Samdani T, Garcia-Aguilar J. Imaging in rectal cancer: magnetic resonance imaging versus endorectal ultrasonography. Surg Oncol Clin N Am. 2014;23(1):59-77.

3. Detering R, van Oostendorp SE, Meyer VM, et al. MRI cT1-2 rectal cancer staging accuracy: a population-based study. $\mathrm{Br} J$ Surg. 2020;107(10):1372-82.

4. Stewart DB, Dietz DW. Total mesorectal excision: What are we doing? Clin Colon Rectal Surg. 2007;20(3):190-202.

5. Smith JJ, Strombom P, Chow OS, et al. Assessment of a watchand-wait strategy for rectal cancer in patients with a complete response after neoadjuvant therapy. JAMA Oncol. 2019;5(4):e185896.

6. Garcia-Aguilar J, Patil S, Kim JK, et al. Preliminary results of the organ preservation of rectal adenocarcinoma (OPRA) trial. $J$ Clin Oncol. 2020;38(15_suppl):4008-4008.

7. van der Valk MJM, Hilling DE, Bastiaannet E, et al. Long-term outcomes of clinical complete responders after neoadjuvant treatment for rectal cancer in the International Watch \& Wait Database (IWWD): an international multicentre registry study. Lancet. 2018;391(10139):2537-45.

8. Garcia-Aguilar J, Renfro LA, Chow OS, et al. Organ preservation for clinical T2N0 distal rectal cancer using neoadjuvant chemoradiotherapy and local excision (ACOSOG Z6041): results of an open-label, single-arm, multi-institutional, phase 2 trial. Lancet Oncol. 2015;16(15):1537-46.

9. Rullier E, Rouanet P, Tuech JJ, et al. Organ preservation for rectal cancer (GRECCAR 2): a prospective, randomised, openlabel, multicentre, phase 3 trial. Lancet. 2017;390(10093):469-79. 
10. Lezoche E, Baldarelli M, Lezoche G, Paganini AM, Gesuita R, Guerrieri M. Randomized clinical trial of endoluminal locoregional resection versus laparoscopic total mesorectal excision for T2 rectal cancer after neoadjuvant therapy. Br $J$ Surg. 2012;99(9):1211-8.

11. Bach SP, Gilbert A, Brock K, et al. Radical surgery versus organ preservation via short-course radiotherapy followed by transanal endoscopic microsurgery for early-stage rectal cancer (TREC): a randomised, open-label feasibility study. Lancet Gastroenterol Hepatol. 2021;6(2):92-105.

12. Barina A, De Paoli A, Delrio P, et al. Rectal sparing approach after preoperative radio- and/or chemotherapy (RESARCH) in patients with rectal cancer: a multicentre observational study. Tech Coloproctol. 2017;21(8):633-40.

13. Garcia-Aguilar J, Smith DD, Avila K, Bergsland EK, Chu P, Krieg RM. Optimal timing of surgery after chemoradiation for advanced rectal cancer: preliminary results of a multicenter, nonrandomized phase II prospective trial. Ann Surg. 2011;254(1):97-102.

14. Haak HE, Žmuc J, Lambregts DMJ, et al. The evaluation of follow-up strategies of watch-and-wait patients with a complete response after neoadjuvant therapy in rectal cancer. Colorectal Dis. 2021;23(7):1785-92.

15. Stijns RCH, de Graaf EJR, Punt CJA, et al. Long-term oncological and functional outcomes of chemoradiotherapy followed by organ-sparing Transanal endoscopic microsurgery for distal rectal cancer: the CARTS Study. JAMA Surg. 2019; 154(1):47-54.

16. van Gijn W, Marijnen CA, Nagtegaal ID, et al. Preoperative radiotherapy combined with total mesorectal excision for resectable rectal cancer: 12-year follow-up of the multicentre, randomised controlled TME trial. Lancet Oncol. 2011;12(6):575-82.

17. Lynn PB, Van der Valk M, Claassen YHM, et al. Chemoradiation and local excision versus total mesorectal excision for T2N0 rectal cancer: comparison of short- and long-term outcomes from two prospective studies. Ann Surg. 2021. https://doi.org/10.1097/ SLA.0000000000005052.

18. Kennecke HF, Brown CJ, Loree JM, et al. CCTG CO.28 primary endpoint analysis: neoadjuvant chemotherapy, excision and observation for early rectal cancer, the NEO trial. J Clin Oncol. 2021;39(15):3508-3508.

19. Rombouts AJM, Al-Najami I, Abbott NL, et al. Can we save the rectum by watchful waiting or transanal microsurgery following (chemo) radiotherapy versus total mesorectal excision for early rectal cancer (STAR-TREC study)?: protocol for a multicentre, randomised feasibility study. BMJ open. 2017;7(12):e019474.

Publisher's Note Springer Nature remains neutral with regard to jurisdictional claims in published maps and institutional affiliations. 